\title{
NOTE ON NORMAL SUBGROUPS OF THE MODULAR GROUP
}

L. GREENBERG

Let $\Gamma$ denote the modular group, namely the group of linear fractional transformations

$$
T(z)=\frac{a z+b}{c z+d},
$$

where $a, b, c, d$ are integers and $a d-b c=1$. It is well known that the transformations

$$
X(z)=-\frac{1}{z}, \quad Y(z)=-\frac{1}{1+z}
$$

generate $\Gamma$, with defining relations

$$
X^{2}=Y^{3}=1 \text {. }
$$

We shall of ten need to consider the element $Z=X Y$, which is a parabolic transformation. Any parabolic transformation in $\Gamma$ is conjugate to a power of $Z$.

Let $N$ be a normal subgroup of finite index $\mu$ in $\Gamma$. The level $n$ of $N$ is defined as the least positive integer such that $Z^{n} \in N$. The conjugacy class of $Z$ in $\Gamma$ splits up into a finite number of equivalence classes under conjugacy by $N$. The number $t$ of equivalence classes is called the parabolic class number of $N$. It is known that the integers $\mu, n, t$ satisfy the relation

$$
\mu=n t .
$$

One way of seeing this relation is the following. $\Gamma$ operates discontinuously in the upper half-plane $D$. We obtain quotient surfaces $S_{\Gamma}=D / \Gamma$ and $S_{N}=D / N$. Since $N$ is a normal subgroup of $\Gamma$, we have a normal (branched) covering $\phi: S_{N} \rightarrow S_{\mathrm{r}} . S_{N}$ is a closed surface with $t$ punctures, and $S_{\Gamma}$ is the sphere with one puncture. If we compactify $S_{\Gamma}$ and $S_{N}$ by adding a point $p$ at the puncture in $S_{\Gamma}$, and points $p_{1}, p_{2}, \cdots, p_{t}$ at the punctures in $S_{N}$, we obtain surfaces $\bar{S}_{\Gamma}, \bar{S}_{N}$ and a normal covering $\bar{\phi}: \bar{S}_{N} \rightarrow \bar{S}_{\Gamma}$. The covering has $\mu$ sheets and there are exactly $t$ points lying over $p$, each of which has branching order $n-1$ (i.e. $n$ sheets meet at each $p_{i}$ ). By counting the sheets over $p$, we find $\mu=n t$.

Received by the editors February 17, 1966. 
It is clear that there is only a finite number of subgroups $N$ of index $\mu$. It is also known that there are infinitely many normal subgroups of level $n$, for all integers $n \geqq 6$. (There are only finitely many for $1 \leqq n \leqq 5$.) To find such groups, one might as well add the relation $Z^{n}=1$ to the relations for $\Gamma$. We then obtain the triangle group $\Delta(2,3, n)$. It is known that this is finite for $n \leqq 5$, and infinite for $n \geqq 6$. In the last case it is known (cf. Fox [1]) that $\Delta(2,3, n)$ contains subgroups of finite index without elements of finite order. It therefore contains infinitely many normal subgroups $N$, such that $Z$ has order $n \bmod N$.

We now ask the analogous question for the parabolic class number t. M. Newman [3] has shown that there are infinitely many nonnormal subgroups of finite index in $\Gamma$ with $t$ parabolic classes, for any integer $t \geqq 1$. He also showed that there are only finitely many normal subgroups with $t \leqq 11$. In this paper we shall show that for any $t \geqq 1$, there is only a finite number of normal subgroups with $t$ parabolic classes.

Let $H$ be a finite homomorphic image of $\Gamma$, and let $F$ be a finite cyclic group. We wish to study extensions $G$ of $F$ by $H$ such that $F$ is contained in the center of $G(H \approx G / F)$. One obtains such extensions from factor sets $\left\{f_{u, v}\right\}$, where $u, v \in H, f_{u, v} \in F$ and

$$
\begin{gathered}
f_{u v, w} f_{u, v}=f_{u, v w} f_{v, w}, \\
f_{u, 1}=f_{1, u}=1 .
\end{gathered}
$$

The extension $G$ then consists of elements $g_{u} f(u \in H, f \in F)$ with multiplication:

$$
\left(g_{u} f_{1}\right)\left(g_{v} f_{2}\right)=g_{u v} f_{u, v} f_{1} f_{2},
$$

(cf. Hall [2]).

Lemma 1. Let $x, y, z$ be the images of $X, Y, Z$ in $H$, and let $z$ have order $k$. Suppose that the factor set $\left\{f_{u, v}\right\}$ satisfies:

(a) $f_{x, x}=f_{y, y} f_{y, y^{2}}=1$,

(b) $f_{z, z} f_{z, z^{2}} \cdots f_{z, z^{k-1}} f_{x, y}^{\mathbf{k}}=f$, where $f$ generates $F$. Then $G$ is a homomorphic image of $\Gamma$.

Proof. Let $\bar{x}=g_{x}, \bar{y}=g_{y}$. Then

$$
\begin{aligned}
& \bar{x}^{2}=g_{x}^{2}=g_{x^{2}} f_{x, x}=1, \\
& \bar{y}^{3}=g_{y}^{3}=g_{y}{ }^{3} f_{y, y} f_{y, y^{2}}=1 .
\end{aligned}
$$

Therefore the subgroup $\bar{G}$ generated by $\bar{x}$ and $\bar{y}$ is a homomorphic image of $\Gamma$. If we can show that $\bar{G} \supset F$, it will follow that $\bar{G}=G$. 
Let $\bar{z}=\bar{x} \bar{y}=g_{x} g_{y}=g_{z} f_{x, y}$. By induction, we can show that $\bar{z}^{r}$ $=g_{z^{r}} f_{z, z} f_{z, z^{2}} \cdots f_{z, z^{r-1}} f_{x, v^{\prime}}^{r}$. In particular $\bar{z}^{k}=g_{z^{k}} f_{z, z} f_{z, z^{2}} \cdots f_{z, z^{k-1}} f_{x, y}^{k}$ $=f$. Therefore $\bar{G} \supset F$ and $\bar{G}=G$.

q.e.d.

Lemma 2. Let $G$ be a finite homomorphic image of $\Gamma$, and let $\bar{x}, \bar{y}, \bar{z}$ be the images of $X, Y, Z$ in $G$. Let $k$ divide the order of $\bar{z}$, and suppose that the subgroup $F$ generated by $\bar{z}^{k}$ is central in $G$. Let $H=G / F$, and let $x, y, z$ be the images of $\bar{x}, \bar{y}, \bar{z}$ in $H$. Then there exists a factor set $\left\{f_{u, v}\right\}$ for $G$, relative to $F$ and $H$, which satisfies conditions (a), (b) of Lemma 1 .

Proof. Choose $\bar{x}, \bar{y}, \bar{y}^{2}, \bar{z}, \bar{z}^{2}, \cdots, \bar{z}^{k-1}$ to be the coset representatives of their $F$-cosets. It will then follow that

$$
f_{x, y}=f_{x, x}=f_{y, y}=f_{y, y^{2}}=f_{z, z}=f_{z, z^{2}}=\cdots=f_{z, z^{k-2}}=1,
$$

and

$$
f_{2, z^{k-1}}=\bar{z}^{k}
$$

q.e.d.

Lemma 3. Let $N$ be a normal subgroup of finite index in $\Gamma$, with $t$ parabolic classes. Let $G=\Gamma / N$ and let $x, y, z$ be the images of $X, Y, Z$ in $G$. Let $U$ be the subgroup generated by $z$. Then $U$ contains a subgroup $F$ such that

(a) $F$ is contained in the center of $G$,

(b) $[G: F] \leqq t^{2}$.

Proof. Let $F=U \cap x U x^{-1}$. Since $F$ is normalized by $x$ and $z$, it is a normal subgroup of $G$. For $g \in G$, let $\alpha_{g}: F \rightarrow F$ be the automorphism $\alpha_{0}(f)=g^{-1} f g$. We then have: $\alpha_{x}^{2}=\alpha_{y}^{3}=1, \alpha_{x} \alpha_{y}=\alpha_{z}=1$. Therefore $\alpha_{x}=\alpha_{y}$ $=1$, so $F$ is central in $G$. Since $[G: U]=\left[G: x U x^{-1}\right]=t$, it follows that $[G: F] \leqq t^{2}$.

q.e.d.

THEOREM 1. Let $N$ be a normal subgroup of $\Gamma$ with $t$ parabolic classes and index $\mu$. Then $\mu \leqq 6 t^{4}$.

Proof. Let $G=\Gamma / N$ and let $\bar{x}, \bar{y}, \bar{z}$ be the images of $X, Y, Z$ in $G$. Let $F$ be the central subgroup from Lemma 3. $F$ is generated by $\bar{z}^{k}$ (where $k$ divides the order of $\bar{z}$ ). Let $H=G / F, r=\operatorname{order}(F), s=$ order $(H)$. Then $\mu=r s$ and $s \leqq t^{2}$.

Let $x, y, z$ be the images of $\bar{x}, \bar{y}, \bar{z}$ in $H$. By Lemma 2 , there is a factor set $\left\{f_{u, v}\right\}$ for $G$, relative to $F$ and $H$, which satisfies conditions (a), (b) of Lemma 1. By a theorem in extension theory (Hall [2], p. 223) the extension $G^{\prime}$ corresponding to the factor set $\left\{f_{u, v}^{s}\right\}$ splits. Since $F$ is central, this means that $G^{\prime} \approx F \times H$.

Let $F^{s}$ denote the subgroup of $F$ consisting of all sth powers, and let $G^{\prime \prime}=\left\{g_{u} f \in G^{\prime} \mid f \in F^{*}\right\}$. $G^{\prime \prime}$ is a subgroup of $G^{\prime}$, since the factors 
$f_{u, 0}^{s} \in F^{s} . G^{\prime \prime}$ is the extension of $F^{s}$ by $H$, corresponding to the factor set $\left\{f_{u, v}^{s}\right\}$. Since the factors $f_{u, v}^{s}$ satisfy conditions (a) and (b) of Lemma 1 (with $F$ replaced by $F^{s}$ ), it follows that $G^{\prime \prime}$ is a homomorphic image of $\Gamma$.

Since $G^{\prime} \approx F \times H$, there is a projection $\pi: G^{\prime} \rightarrow F$. Let $F^{\prime}=\pi\left(G^{\prime \prime}\right)$. $F^{\prime}$ is a homomorphic image of $\Gamma$, which contains $F^{s}$. Since an abelian homomorphic image of $\Gamma$ has order $\leqq 6$, we have $\left|F^{*}\right| \leqq\left|F^{\prime}\right| \leqq 6$. But $r / s \leqq r /(r, s)=\left|F^{*}\right|$. Therefore $r \leqq 6 s, s \leqq t^{2}$ and $\mu=r s \leqq 6 t^{4}$. q.e.d.

COROLLARY. There are only a finite number of normal subgroups of $\Gamma$ with $t$ parabolic classes.

TheоReм 2. Let $p$ be a prime such that $p \equiv-1(\bmod 3)$. Then there is no normal subgroup of finite index in $\Gamma$ with $p$ parabolic classes. ${ }^{1}$

Proof. Suppose $N$ is a normal subgroup with $p$ parabolic classes, level $n$ and index $\mu$. By Lemma $3, G=\Gamma / N$ contains a central subgroup $F$, generated by $z^{k}$ (where $k$ divides $n$ ) such that $[G: F] \leqq p^{2}$. $H=G / F$ is a quotient group of $\Gamma: H=\Gamma / M$. Since $z$ has order $k$ in $H, M$ has level $k$, and index $\nu \leqq p^{2}$. Since $|F|=n / k, \nu=k \mu / n=k p$, so that $M$ also has $p$ parabolic classes. The new level $k$ satisfies $k \leqq p$. If $k=p$ then $H$ is a group of order $\nu=p^{2}$, and therefore $H$ is abelian. But all abelian quotient groups of $\Gamma$ have order $\leqq 6$, therefore $\nu<p^{2}$ and $k<p$.

Let $S$ be a Sylow $p$-subgroup of $H$ and let $m$ be the number of conjugates of $S$ in $H$. Then $m$ is of the form $m=1+p r$ and $m \mid k p$. Since $k<p$, it follows that $m=1$ and $S$ is normal in $H$.

Let $K$ be the subgroup generated by $z \in H . K \cap S$ has order dividing $|K|=k$ and $|S|=p$. Since these are relatively prime, $K \cap S=\{1\}$. Therefore $H$ is the semidirect product $H=S \cdot K$. Thus $K$ is a homomorphic image of $H$, and therefore of $\Gamma$. Since $K$ is abelian, the order $k \leqq 6$. Thus $k=1,2,3$ or 6 . However, $6 \mid \nu=k p$, since $x$ and $y$ are nontrivial in $H$ (if $x=1$ or $y=1$ then $\nu \leqq 3$ ). Thus $6 \mid k, k=6$ and $\nu=6 p$. But M. Newman [3] showed that $6 p$ does not occur as the index of a normal subgroup of $\Gamma$, when $p \equiv-1(\bmod 3)$ and $p$ is prime. q.e.d.

\section{REFERENCES}

1. R. Fox, On Fenchel's conjecture about F-groups, Mat. Tids. B (1952), 61-65.

2. M. Hall, The theory of groups, Macmillan, New York, 1959.

3. M. Newman, Classification of normal subgroups of the modular group (to appear).

UNIVERSITY OF MARYLAND AND

National Bureau of Standards

1 This theorem was proved simultaneously and independently by M. Newman. 